\title{
A Phase II study of S-I plus oral leucovorin in heavily treated metastatic colorectal cancer patients
}

This article was published in the following Dove Press journal:

Cancer Management and Research

\author{
Hung-Chih Hsu',2 \\ Wen-Chi Chou ${ }^{1,2}$ \\ Feng-Che Kuan ${ }^{2,3}$ \\ Kuan-Der Lee ${ }^{4}$ \\ Kun-Ming $\operatorname{Rau}^{2,5}$ \\ Jen-Seng Huang ${ }^{2,6}$ \\ Tsai-Sheng Yang ${ }^{1,2}$
}

'Division of Hematology and Oncology, Department of Internal Medicine, Chang Gung Memorial Hospital at Linkou, Guishan, Taoyuan, Taiwan, Republic of China; ${ }^{2}$ College of Medicine, Chang Gung University, Tao-Yuan, Taiwan, Republic of China; ${ }^{3}$ Division of Hematology and Oncology, Chang Gung Memorial Hospital, Chiayi, Puzi City, Taiwan, Republic of China; ${ }^{4}$ Division of Hematology and Oncology, Department of Medicine, Taipei Medical University Hospital and Taipei Medical University, Taipei, Taiwan, Republic of China; ${ }^{5}$ Division of Hematology and Oncology, Chang Gung Memorial Hospital, Kaohsiung, Taiwan, Republic of China; ${ }^{6}$ Division of Hematology and Oncology, Chang Gung Memorial Hospital, Keelung, Taiwan, Republic of China
Correspondence: Tsai-Sheng Yang Division of Hematology and Oncology, Department of Internal Medicine, Chang Gung Memorial Hospital at Linkou, No 5, Fu Hsing Street, Kuei Shan, Taoyuan County, Taiwan 33305, Republic of China Tel +886 $3328 \quad 1200$ ext 8825

Fax +8863328 I200 ext 2362 Email a48I I24@cgmh.org.tw
Purpose: Fewer treatment options are available for refractory metastatic colorectal cancer (mCRC). In early trials, S-1 monotherapy was effective for mCRC patients after chemotherapy failure and its combination with oral leucovorin therapy offers promising results in untreated mCRC. Hence, we conduct a Phase II trial to assess the efficacy of S-1 plus oral leucovorin (SL) in refractory $\mathrm{mCRC}$ that progressed after multiple prior standard therapies.

Methods: In this open-label, single-arm study, we enrolled the refractory mCRC patients who received fluoropyrimidine, oxaliplatin, and irinotecan treatment and at least one targeted therapy previously. The doses of SL were 40-60 and $30 \mathrm{mg}$ twice daily separately. They were administered for 7 days in a 2-week cycle. Treatment was continued until disease progression. Results: Of the 41 enrolled patients, 36 patients were evaluable with $61.1 \%$ disease control rate. The median progression-free survival and overall survival were 2.55 and 7.63 months, respectively. Regression change in tumor size stayed $10 \%-20 \%$ in five patients (13.9\%) through 18 weeks after treatment, and two patients continued free from tumor progression at 30 and 42 weeks. Compared with moderate heavily pretreated mCRC patient subgroup ( $\leq 4$ prior regimens), the severe heavily pretreated subgroup ( $\geq 5$ prior regimens) showed similar disease control rate and survival benefit. Grade 3 or higher toxicities were documented only in 11 patients (26.8\%).

Conclusion: SL shows potential as a salvage regimen in refractory $\mathrm{mCRC}$ patients especially in the severe heavily pretreated setting and is well tolerated in these patients.

Keywords: refractory metastatic colorectal cancer, S-1, oral leucovorin, Phase II, survival and safety

\section{Introduction}

Colorectal cancer (CRC) remains one of the most common types of cancer and leading causes of cancer death worldwide. Indeed, despite improvements in diagnostic and treatment modalities, more than 1 million people develop CRC each year, with over 600,000 patients dying of the disease annually. It is the most prevalent form of cancer among Taiwanese, topping the list of the most common cancers in Taiwan since 2006.

About $50-60 \%$ of patients diagnosed with CRC develop metastatic CRC (mCRC), and $80-90 \%$ of these patients have unresectable metastatic disease, which has been the major cause of death in this population. In the past decades, major advances in systemic therapy (including chemotherapy and biological therapy) for mCRC have allowed these patients to survive for $>2$ years. ${ }^{1,2}$

Three types of cytotoxic drugs for the treatment of mCRC included fluoropyrimidines (eg, 5-fluorouracil [5-FU] and capecitabine), oxaliplatin, and irinotecan. A study of 6,286 patients from nine trials evaluated the benefits and risks associated 
with intensive first-line $\mathrm{mCRC}$ treatment with these cytotoxic drugs, and the result showed that these treatments have similar therapeutic efficacy for patients. ${ }^{3-12}$ More recently, new therapeutic approaches called targeted therapies were added to the chemotherapies based on the information described earlier. Anti-vascular endothelial growth factor (VEGF) agent, bevacizumab, and anti-EGFR agents, cetuximab and panitumumab, are administered in combination with chemotherapy regimen for mCRC. ${ }^{13}$

Currently, no standard treatment exists for patients with mCRC who have progressed after treatment with 5-FU, oxaliplatin, irinotecan, anti-EGFR/VEGF agents, and regorafenib. The efficacy and safety of further treatment in heavily treated patients with $\mathrm{mCRC}$ should be investigated.

From previous studies, ${ }^{14-17} \mathrm{~S}-1$, an oral fluoropyrimidine combining tegafur with the two such as modulators gimeracil and oteracil, demonstrates good efficacy with manageable toxicities as monotherapy as well as combination therapy in mCRC patients who were previously treated with chemotherapy. For S-1 monotherapy, the objective response rate (ORR) was approximately 15\%, disease control rate (DCR) was above $40 \%$, and time to tumor progression (TTP) was about 2-3 months. ${ }^{14,15}$ For S-1 combination therapy with irinotecan or irinotecan plus oxaliplatin, the ORR was above $20 \%$, DCR was above $50 \%$, and progression-free survival (PFS) varied from 2.6 to 5.8 months for mCRC patients previously treated with chemotherapy. ${ }^{16,17}$

One of the components of S-1, tegafur, is a prodrug of 5-FU. 5-FU is usually administered with leucovorin (LV) in $\mathrm{mCRC}$, which can enhance the therapeutic and toxic effects of 5-FU. ${ }^{18}$ The combination of S-1 and LV (SL) is known to potentiate the antitumor activity of CRC in preclinical studies. ${ }^{19}$ Through the mechanism of calcium folinate amplifying the efficacy of 5-FU in patients with mCRC, we expect that the addition of calcium folinate will enhance the efficacy of S-1. Comparison between two early Phase II trials in untreated $\mathrm{mCRC}$ showed that SL (same as calcium folinate) demonstrated better clinical benefit than S-1 monotherapy. ${ }^{20,21}$ In a Phase II trial of SL in patients with previously untreated $\mathrm{mCRC}, \mathrm{S}-1$ was given orally twice daily for 2 consecutive weeks at a daily dose of 80-120 mg, followed by a 2 -week rest period, within a 4 -week cycle. LV was given orally twice a day at a daily dose of $50 \mathrm{mg}$, simultaneously with S- $1 .{ }^{20}$ Of the 56 patients, 32 (57\%) patients had partial response (PR). The median TTP was 6.7 months (95\% CI, 5.4-7.9), and the median OS was 24.3 months. However, $32 \%$ patients had grade 3 diarrhea $^{20}$ that might not be tolerated in salvage setting.
Thus, we conducted a Phase II trial to assess the efficacy and safety of SL as a salvage therapy in refractory mCRC patients who failed both chemotherapy and anti-EGFR/ VEGF agents. We also modified the regimen from 14 days dosing in a 28-day cycle to 7 days dosing in a 14-day cycle intending to limit the toxicity while maintaining the same monthly dose for efficacy.

\section{Methods \\ Study design}

This Phase II open-label, single-arm study was to determine the DCR of combination of SL in heavily pretreated mCRC patients. Patients aged 20 years or older were enrolled in the study. The eligibility criteria were as follows: metastatic or unresectable colorectal cancer, histologically proven adenocarcinoma with the presence of at least one measurable tumor lesion, previously treated with fluoropyrimidine, oxaliplatin, and irinotecan, and targeted therapy with bevacizumab and cetuximab in case of KRAS wild type, Eastern Cooperative Oncology Group (ECOG) performance status of 0-2, and adequate organ functions. Patients with the presence of brain metastasis, the life expectancy of less than 12 weeks, and other malignancies were excluded. This study was conducted in accordance with the Declaration of Helsinki and the International Conference on Harmonization guidelines for Good Clinical Practice. The clinical study protocol was approved by the institutional review board of Chang Gung Memorial Hospital (IRB 100-4279B). Details of the TTYTG1307 study design have been registered via ClinicalTrials.gov with the identifier NCT03517618. All patients provided written informed consent prior to study participation.

The primary objective is to determine DCR, the sum of complete response (CR) + PR + stable disease (SD), of SL in patients with heavily pretreated $\mathrm{mCRC}$. The secondary objectives include determining ORR, PFS, TTP, overall survival (OS), and safety profile.

Patients were categorized into intend-to-treat (ITT), per-protocol (PP), and safety population according to the following definitions: ITT was defined as all registered subjects who received at least one dose of study treatment without major protocol violations, such as noncompliance with the eligibility criteria, or other major violations (defined prior to data lock) during the study. PP population was defined as the subset of the ITT population and subjects who complete 2 cycles of study treatment and obtain first scheduled tumor assessment post-treatment at least. The subject with confirmed early disease progression would be included in PP population. PP population has evaluable patients. 
Tumor response was evaluated according to the Response Evaluation Criteria Solid Tumors (RECIST) version 1.1. CT scan was performed within 28 days prior to treatment, and then, every 6 weeks from the start of treatment or performed for the recurrence developed during the study (including clinical suspicion). The allowable window for scheduling imaging studies was \pm 1 week. Analysis for the DCR and other secondary endpoints was conducted on both the ITT and PP populations.

TTP was measured from the start date of study treatment to the date of disease progression. PFS was calculated from the start date of study treatment until objective disease progression or death due to any cause. OS was measured from the start date of study treatment to the date of death. TTP, PFS, and OS were evaluated by Kaplan-Meier method, and the analysis was carried out in descriptive statistics, presented by point estimate and $95 \% \mathrm{CI}$ for the efficacy variable. Basic demographic data were summarized as $\mathrm{n}(\%)$ for categorical variables and median with range for continuous variables. All statistical tests were two sided, and $P<0.05$ was considered statistically significant.

Hematology and biochemistry assessments were performed within 14 days before registration and at each clinical visit. The incidence and percentage of patients with at least one occurrence of a preferred term were included, according to the most severe National Cancer Institute-Common Terminology Criteria for Adverse Events (NCI-CTCAE) version 4.0 grade. Laboratorial toxicity was presented according to their worse NCI-CTCAE grade by cycle.

\section{Treatment}

Patients enrolled in the study were treated with S-1 (Taiho Pharmaceutical Company Ltd., Tokyo, Japan) in combination with calcium folinate (TTY Biopharm Company Ltd.). Patients received S-1 orally twice daily at a dose according to the body surface area (BSA) $\left(<1.25 \mathrm{~m}^{2}, 80 \mathrm{mg} /\right.$ day; $\geq 1.25$ to $<1.5 \mathrm{~m}^{2}, 100 \mathrm{mg} /$ day; $\geq 1.5 \mathrm{~m}^{2}, 120 \mathrm{mg} /$ day) and LV orally twice daily at $30 \mathrm{mg} /$ day on days $1-7$ of a 14-day cycle. Treatment was repeated every 2 weeks and administered until disease progression, intolerable toxicity, or consent withdrawal. The dose was reduced to one or two levels; each level has the reduction of $20 \mathrm{mg}$ of S-1 per day. In brief, patients with the initial dose of $120 \mathrm{mg}$ /day have the reduction level (one level) of $100 \mathrm{mg} /$ day and the reduction level (two levels) of $80 \mathrm{mg} /$ day. In patients with grade 3 or greater hematological and nonhematological toxicity for the first time, the dose of S-1 was reduced by one level $(20 \mathrm{mg} /$ day). In the patients with grade 3 or greater hematological and nonhematological toxicity for the second time, the dose of S-1 was reduced by two levels $(40 \mathrm{mg} /$ day). Patients who require more than two dose reduction level were rejected from the study. If the patient has not recovered after a 2-week delay, consideration should be given to discontinuing therapy. No dose modification for calcium folinate was required unless S-1 was discontinued, and then, calcium folinate should be discontinued as well.

Dose modification after the first treatment was made based on the worst toxicity degree graded by the common terminology criteria for adverse events of cancer therapy evaluation program of NCI-CTCAE version 4.0. For those toxicities considered by the investigator to be unlikely to become serious or life threatening and that do not result in a delay or interruption of therapy (eg, alopecia and altered taste), treatment was continued at the same dose without reduction or interruption. No dose reductions or interruptions were required for anemia as this can be satisfactorily controlled by transfusions.

Before the start of subsequent cycles of therapy, patient should meet all the starting criteria described below, otherwise the treatment should be delayed until recover to the same criteria within 2 weeks. The starting criteria for starting subsequent cycle are hemoglobin greater or equal to $9 \mathrm{~g} / \mathrm{dL}$, absolute neutrophil greater or equal to $1,500 / \mathrm{mm}^{3}$, platelet count greater or equal $100,000 / \mathrm{mm}^{3}$, total bilirubin greater or equal to two times upper limit of normal (ULN), aspartate transaminase (AST)/alanine transaminase (ALT) greater or equal to three times ULN or return to baseline, diarrhea and stomatitis greater or equal to grade 1, skin rash greater or equal to grade 3 , and other nonhematological toxicities greater or equal to grade 1 or return to baseline.

\section{Results Patients' characteristics}

Between August 2014 and December 2014, 41 heavily pretreated metastatic colorectal patients were enrolled from four branches of Chang-Gung Memorial Hospital in Taiwan. As of the last date of follow-up on September 10, 2015, four patients were withdrawn from the study prematurely, leaving 36 patients in PP population with 41 patients in ITT. Baseline characteristics are summarized in Table 1 . The median age was 62 years (range $40-87$ years). Twenty-six patients were male and 15 patients were female. Most patients had ECOG 0 or $1(39,95.1 \%)$. The most common site of metastatic was liver in 31 patients (75.6\%), KRAS wild type and mutations were presented in 22 patients (53.7\%) and 19 patients (46.3\%), respectively, and $20(48.8 \%)$ patients had 
Table I Baseline patient characteristics $(N=4 I)$

\begin{tabular}{|c|c|}
\hline Characteristics & n (\%) \\
\hline \multicolumn{2}{|l|}{ Age (years) } \\
\hline Mean \pm SD & $63.4 \pm 10.4$ \\
\hline Median (minimum-maximum) & $62(40-87)$ \\
\hline \multicolumn{2}{|l|}{ Gender } \\
\hline Male & $26(63.4 \%)$ \\
\hline Female & $15(36.6 \%)$ \\
\hline \multicolumn{2}{|l|}{ ECOG performance status } \\
\hline 0 & $19(46.3 \%)$ \\
\hline $\mathrm{I}$ & $20(48.8 \%)$ \\
\hline 2 & $2(4.9 \%)$ \\
\hline \multicolumn{2}{|l|}{ Metastatic sites } \\
\hline Liver & $31(75.6 \%)$ \\
\hline Lung & $20(48.8 \%)$ \\
\hline Lymph node & $4(9.8 \%)$ \\
\hline Ovary & $\mathrm{I}(2.4 \%)$ \\
\hline Others & $13(31.7 \%)$ \\
\hline \multicolumn{2}{|l|}{ KRAS mutation } \\
\hline Yes & $19(46.3 \%)$ \\
\hline No & $22(53.7 \%)$ \\
\hline \multicolumn{2}{|l|}{ Lines of prior anticancer therapies } \\
\hline Range (minimum-maximum) & $4(2-9)$ \\
\hline 2 & $3(7.3 \%)$ \\
\hline 3 & $10(24.4 \%)$ \\
\hline$\geq 4$ & $28(68.3 \%)$ \\
\hline \multicolumn{2}{|l|}{ Prior systemic anticancer agents } \\
\hline Fluoropyrimidines & $41(100 \%)$ \\
\hline Irinotecan & $41(100 \%)$ \\
\hline Oxaliplatin & $41(100 \%)$ \\
\hline Bevacizumab & $41(100 \%)$ \\
\hline Anti-EGFR monoclonal antibody & $22(53.7 \%)$ \\
\hline Regorafenib & $\mathrm{I}(2.4 \%)$ \\
\hline
\end{tabular}

Abbreviation: ECOG, Eastern Cooperative Oncology Group.

been treated $\geq 5$ lines of chemotherapy. Of the 41 enrolled patients, five patients were early withdrawn from study and received study medications less than 2 weeks. Among the early withdrawn subjects, three of them refused study medication due to adverse events (mucositis, vomiting, and abdominal distention, respectively), one subject withdrew under investigator's discretion due to ileus, and one subject withdrew due to rapid progression of disease.

\section{Efficacy results}

Treatment results on efficacy data are shown in Table 2 and Figure 1. The DCR of SL is $61.1 \%$ (95\% CI, 43.5-76.9). Of the 41 enrolled patients, five patients were not evaluable, and of the 36 evaluable patients (PP population), 22 patients (61.1\%) had SD. No patient had PR or CR. The median PFS was 2.55 months ( $95 \% \mathrm{CI}, 1.37-2.83$ ), and the median OS was 7.63 months (95\% CI, 6.17-9.07) in evaluable patients (PP). In ITT, the median PFS was 2.50 months $(95 \% \mathrm{CI}$,
Table 2 Tumor response and dosing parameters (evaluable, $\mathrm{N}$ =36)

\begin{tabular}{|c|c|c|}
\hline Parameters & n (\%) & $P$-value \\
\hline \multicolumn{3}{|l|}{ Best response } \\
\hline CR or $\mathrm{PR}$ & $0(0.0 \%)$ & \\
\hline SD & $22(61.1 \%)$ & \\
\hline PD & $14(38.9 \%)$ & \\
\hline NE & $0(0.0 \%)$ & \\
\hline DCR (\%) & $61.1 \%$ & \\
\hline $95 \% \mathrm{Cl}$ & $43.5-76.9$ & \\
\hline \multicolumn{3}{|l|}{ DCR by KRAS mutation status } \\
\hline KRAS mutant $(n=17)$ & $64.7 \%(11 / 17)$ & \\
\hline KRAS wild type $(n=19)$ & $57.9 \%(11 / 19)$ & 0.742 \\
\hline \multicolumn{3}{|c|}{ DCR by lines of prior treatments } \\
\hline$\leq 4$ lines $(n=18)$ & $61.1 \%(11 / 18)$ & \\
\hline$\geq 5$ lines $(n=18)$ & $61.1 \%(11 / 18)$ & 1.000 \\
\hline Median PFS (months) & 2.55 & \\
\hline $95 \% \mathrm{Cl}$ & $1.37-2.83$ & \\
\hline Median OS (months) & 7.63 & \\
\hline $95 \% \mathrm{Cl}$ & $6.17-9.07$ & \\
\hline \multicolumn{3}{|c|}{ Number of TS-I cycles received } \\
\hline Median (IQR) & $5.5(3-7)$ & \\
\hline Range (minimum-maximum) & $|-2|$ & \\
\hline Sum of total cycles & 211 & \\
\hline \multicolumn{3}{|c|}{ Dose intensity (\% of planned dose) } \\
\hline Mean \pm SD & $90.1 \pm 13.2$ & \\
\hline Median (IQR) & $99.5(81.7,100)$ & \\
\hline \multicolumn{3}{|c|}{ Subsequent therapy after TS-I/LV } \\
\hline Chemotherapy & $20(61.0 \%)$ & \\
\hline Regorafenib & $3(10.0 \%)$ & \\
\hline Investigational product & I (2.4\%) & \\
\hline
\end{tabular}

Abbreviations: $C R$, complete response; $D C R$, disease control rate; $L V$, leucovorin; $\mathrm{NE}$, not evaluable; OS, overall survival; $\mathrm{PD}$, progressive disease; $\mathrm{PR}$, partial response; PFS, progression-free survival; SD, stable disease.

1.37-2.83) and the median OS was 7.63 months (95\% CI, 5.80-9.07) (Figure S1). DCR of SL in KRAS mutant or wild type is similar in both (ITT and PP populations $(P=0.742$, data not shown). Percentage of change in target lesion size stayed below 20 compared to baseline in five patients $(5 / 36$, $13.9 \%)$ through 18 weeks after treatment, with two patients continued free from tumor progression at 30 and 42 weeks after the treatment (Figure 2). Compared with moderate heavily pretreated $\mathrm{mCRC}$ patient subgroup (number of prior regimens $\leq 4, n=18$ ), the severe heavily pretreated subgroup (number of prior regimens $\geq 5, n=18$ ) could have well DCR and survival benefit (DCR: $\geq 5$ lines group vs $\leq 4$ lines group $=61.1$ vs $61.1 \%, P=1.000 ;$ PFS: $\geq 5$ lines group vs $\leq 4$ lines group $=2.48$ vs 2.62 months, $P=0.532$; OS: $\geq 5$ lines group vs $\leq 4$ lines group $=7.6$ vs 8.2 months, $P=0.622$ ). PFS and OS of SL between patient subgroups with positive KRAS mutation and negative KRAS mutation are also similar (Figure 3). 

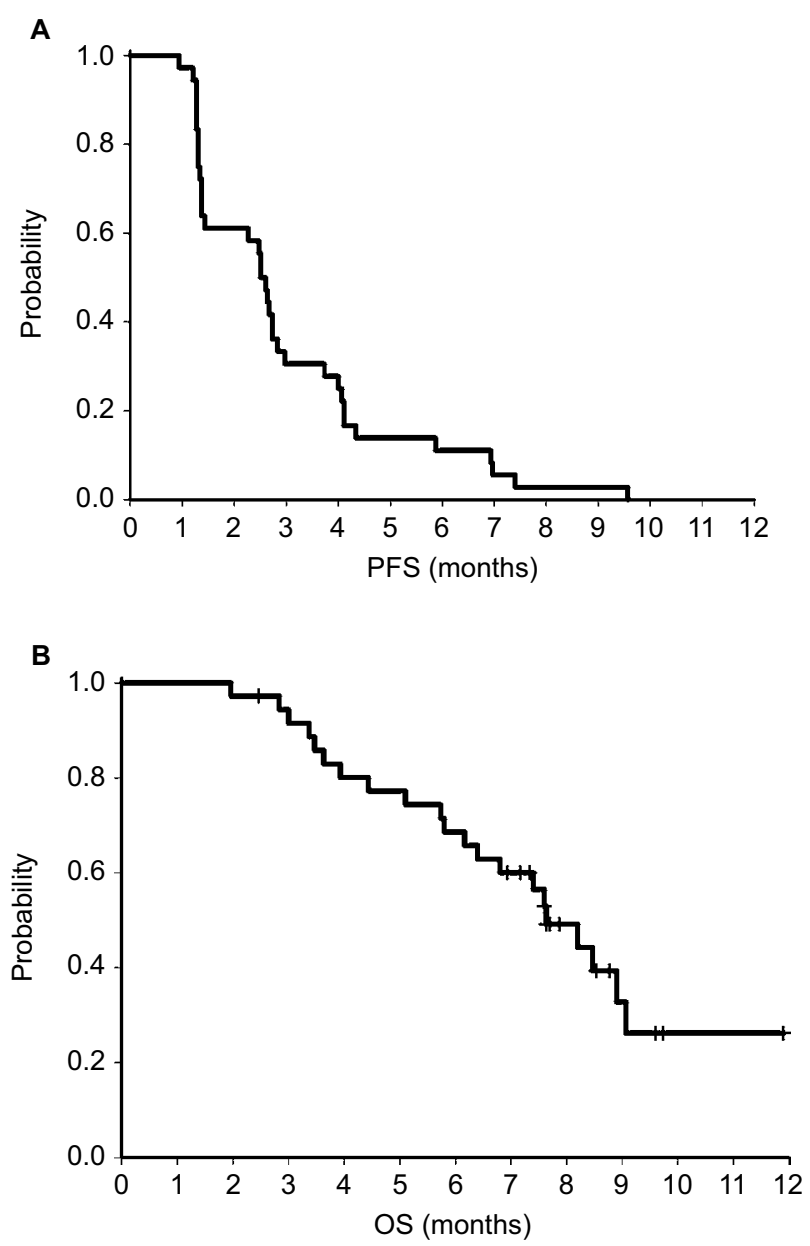

Figure I Kaplan-Meier survival curve for PFS (A) and OS (B) in the PP population $(n=36)$.

Notes: The median PFS was 2.55 months ( $95 \% \mathrm{Cl}$, I.37-2.83). The median OS was 7.63 months ( $95 \% \mathrm{Cl}, 6.17-9.07)$.

Abbreviations: OS, overall survival; PFS, progression-free survival; PP, per-protocol.

\section{Dose reduction and intensity}

For PP population, the total number of treatment cycles administered was 211 and the median number of cycles administered was 5.5 (range 1-21, IQR 3-7). The relative dose intensity to the planned dose was $90.1 \%$ for SL. Dose reduction was required in seven patients (17\%). The most common causes of dose reduction were grade 3 mucositis. Currently, all patients discontinued treatment: 35 (97\%) patients because of disease progression and one (3\%) patient because of consent withdrawal. Twenty-four patients received subsequent treatment after the study, including three patients receiving regorafenib, 20 patients receiving chemotherapy, and one patient receiving a new investigational drug (Table 2).

\section{Safety results}

Adverse effect data are summarized in Table 3. Nonhematological toxicities were more common than hematological toxicities. The most common nonhematological toxicities of any grade were stomatitis (46.3\%), skin hyperpigmentation $(46.3 \%)$, diarrhea $(36.6 \%)$, anorexia $(34.2 \%)$, nausea (29.3\%), hand-foot syndrome (26.8\%), fatigue (24.4\%), and vomiting $(22.0 \%)$. Only one patient with grade 4 mucositis was observed. Grade 3 toxicities were documented in nine patients (24.3\%). Incidence of most frequent grade 3/4 toxicities was stomatitis/mucositis (14.6\%), bilirubin increased (4.9\%) and AST increased, anorexia, nausea, vomiting, fatigue, and hand-foot syndrome each with (2.4\%). No grade $3 / 4$ diarrhea or hematological toxicities were observed.

\section{Discussion}

Although the present chemotherapy (including 5-FU with irinotecan and oxaliplatin) and targeted therapy (cetuximab and bevacizumab) have proven effective in the treatment of $\mathrm{mCRC}$ as first-line and second-line treatments, few reports of salvage therapy provided satisfactory results in heavily treated patients. Oral agents could be a promising alternative with regard to these patients' general condition and quality of life. The present study first demonstrated that SL has significant clinical activity, yielding $61.1 \%$ of DCR in refractory $\mathrm{mCRC}$ patients. Even in the severe heavily treated mCRC patients ( $\geq 5$ lines of prior therapy), the regimen also resulted in $61.1 \%(22 / 36)$ of DCR. This trial also achieved a favorable median OS of 7.63 months.

Fewer drugs (including regorafenib and TAS-102) had been proved efficacy in the setting of refractory $\mathrm{mCRC}$ and potent new anti-cancer agents/new combination therapy have been developed in recent years. ${ }^{22-24}$ In a Phase III study, the DCR of regorafenib group was $51 \%$, with slightly improved OS with regorafenib than with placebo (median OS 8.8 months [95\% CI, 7.3-9.8] in the regorafenib group vs 6.3 months [95\% CI, 4.8-7.6] in the placebo group). ${ }^{23}$ In the Phase III randomized pivotal study of TAS-102, the median OS improved was 7.1 months with TAS-102, with a significantly lowered HR for death in the TAS-102 group vs the placebo group at $0.68(95 \% \mathrm{CI}, 0.58-0.81 ; P<0.001) .{ }^{24}$ Disease control $(\mathrm{CR}+\mathrm{PR}+\mathrm{SD})$ was achieved at $44 \%$ in the TAS group vs only $16 \%$ in the placebo group. ${ }^{24}$ Evidence is growing for the feasibility of anti-EGFR antibody rechallenge in patients with baseline KRAS wild-type mCRC whose disease progresses on first-line anti-EGFR therapy plus chemotherapy. ${ }^{25}$ Santini et $\mathrm{al}^{26}$ reported that 39 patients with KRAS wild-type mCRC were rechallenged with cetuximab plus irinotecan after a median treatment break at 6 months and showed that astonishing results with cetuximab plus irinotecan rechallenge at $53.8 \%$ ORR. Overall, DCR was further taken up to $89.7 \%(C R+P R+S D)$. The median PFS 


\section{A}

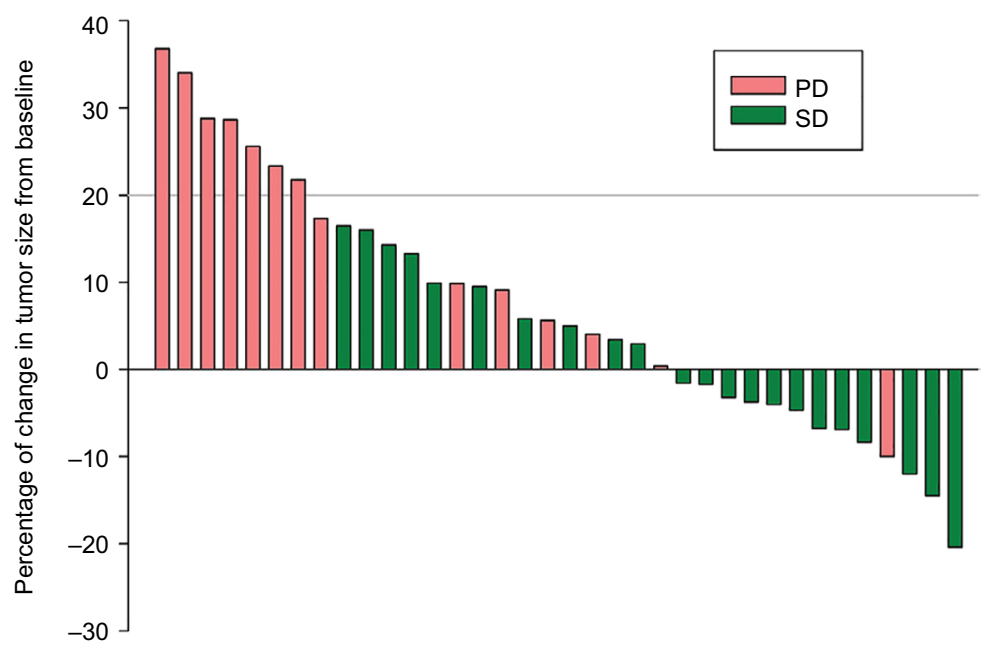

B

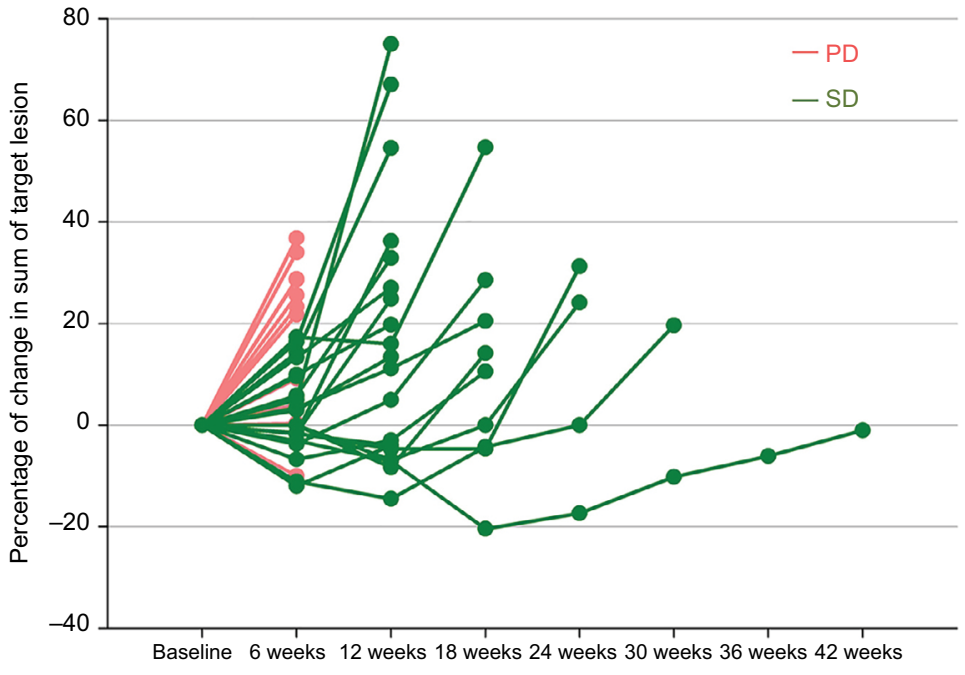

Figure 2 (A) Waterfall plots of best overall percentage of change from baseline in sum of target lesion diameters. (B) Spider plots of percentage of change in sum of target lesion diameters by subject.

Note: Evaluation was assessed by the site investigators (evaluable patients only, $n=36$ )

Abbreviations: PD, progressive disease; SD, stable disease.

was achieved at 6.6 months. In a recent prospective study (the CRICKET study), 27 patients with mCRC were treated with cetuximab plus FOLFIRI or FOLFOXIRI in the first line followed by bevacizumab plus oxaliplatin-based chemotherapy in the second line and cetuximab plus irinotecan rechallenge in the third line. The third-line ORR was $23 \%$, with $54 \%$ of patients having disease control. ${ }^{27}$ Thus, from these studies, rechallenge with anti-EGFR antibody therapy had more DCR and was well tolerated. Compared with these two drugs (regorafenib and TAS-102), our study showed that SL had similar PFS and OS in refractory mCRC patients.

The clinical efficacy of 5-FU against malignancies is well known to be potentiated by adding calcium folinate (LV).
As compared with 5-FU alone, the response rates and OS in patients with advanced CRC are improved when combined 5 -FU with calcium folinate as shown in a meta-analysis consisting of $>3,000$ patients' clinical data. ${ }^{18}$ Likewise, combinations of oral fluoropyrimidine-based regimen (UFT) and LV have been shown to be as effective as intravenous infusion of 5-FU with LV, with favorable safety profile against previously untreated mCRC, ${ }^{28,29}$ as well as in adjuvant setting in patients with curatively resected stage II/III colon cancer. ${ }^{30}$ The addition of LV enhanced the antitumor activity of S-1 by TS inhibition. In a 4-week cycle, compared with S-1 monotherapy for previously untreated $\mathrm{mCRC}$, SL combination therapy demonstrated an improvement in ORR from 35\% 
Table 3 Treatment-related adverse events $(\mathrm{N}=4 \mathrm{I})$

\begin{tabular}{|c|c|c|c|c|c|c|}
\hline \multirow[t]{2}{*}{ Adverse event terms } & \multicolumn{2}{|c|}{ Total } & \multicolumn{2}{|l|}{ G3 } & \multicolumn{2}{|c|}{ G4 } \\
\hline & $\mathbf{n}$ & $\%$ & $\mathbf{n}$ & $\%$ & $\mathbf{n}$ & $\%$ \\
\hline Any of the following & 37 & 90.24 & 11 & 26.83 & $\mathrm{I}$ & 2.44 \\
\hline Skin hyperpigmentation & 19 & 46.34 & & & & \\
\hline Mucositis/stomatitis & 19 & 46.34 & 5 & 12.2 & $\mathrm{I}$ & 2.44 \\
\hline Diarrhea & 15 & 36.59 & & & & \\
\hline Anorexia & 14 & 34.15 & 1 & 2.44 & & \\
\hline Nausea & 12 & 29.27 & $\mathrm{I}$ & 2.44 & & \\
\hline Hand-foot syndrome & 11 & 26.83 & $\mathrm{I}$ & 2.44 & & \\
\hline Fatigue & 10 & 24.39 & $\mathrm{I}$ & 2.44 & & \\
\hline Vomiting & 9 & 21.95 & $\mathrm{I}$ & 2.44 & & \\
\hline Maculo-papular rash & 8 & 19.51 & & & & \\
\hline Body weight loss & 8 & $19.5 \mid$ & & & & \\
\hline Dyspnea & 7 & 17.07 & & & & \\
\hline $\begin{array}{l}\text { Abdomen discomfort/ } \\
\text { distension }\end{array}$ & 7 & 17.07 & 1 & 2.44 & & \\
\hline Anemia & 6 & 14.63 & & & & \\
\hline Bilirubin increased & 6 & 14.63 & 2 & 4.88 & & \\
\hline Edema & 6 & 14.63 & & & & \\
\hline Pain - abdomen & 6 & 14.63 & $\mathrm{I}$ & 2.44 & & \\
\hline Pain - back & 6 & 14.63 & $\mathrm{I}$ & 2.44 & & \\
\hline Pruritus & 5 & 12.2 & & & & \\
\hline Constipation & 5 & 12.2 & & & & \\
\hline Pain - chest & 5 & 12.2 & & & & \\
\hline Fever & 5 & 12.2 & & & & \\
\hline AST increased & 4 & 9.76 & $\mathrm{I}$ & 2.44 & & \\
\hline Creatinine increased & 4 & 9.76 & & & & \\
\hline
\end{tabular}

Abbreviation: AST, aspartate transaminase.

to $57 \%$ and an improvement in time to progression from 5.3 to 6.7 months. ${ }^{20,21}$

The use of SL has been well studied in chemo-naive mCRC patients. Li et al ${ }^{31}$ recently published the results of a single-arm Phase II study of 1-week on 1-week off regimen in patients with chemo-naive mCRC from Japan and China. Of the 71 eligible patients, the response rate was $53.5 \%$ and the DCR was $83.1 \%$. The median PFS and OS were 6.5 and 24.3 months, respectively. The incidences of grade 3 toxicities were diarrhea $8.3 \%$, anorexia $2.8 \%$, stomatitis $8.3 \%$, and neutropenia $9.7 \%$. The adverse events are well tolerated that was similar to that of the regimen in our study even in different therapy settings.

Furthermore, several studies reported that mechanism of resistance to 5-FU is related to increased thymidylate synthase (TS) expression and 5-FU-resistant cell lines show increased TS mRNA expression, protein expression, and activity in in vitro and in vivo assays. ${ }^{32,33} \mathrm{~S}-1$ could have antitumor activity in chemoresistant cancer cells in vitro via inhibiting TS. ${ }^{34}$ Thus, combination of SL may have contributed in overcoming resistance to 5-FU. However, prior study with SL in salvage setting on heavily treated $\mathrm{mCRC}$ patients is scarce before the current study. Lee et $\mathrm{al}^{14}$ and Jeung et $\mathrm{al}^{15}$ conducted studies in mCRC patients who previously treated with two or three lines of standard chemotherapy regimens before receiving $\mathrm{S}-1$ as mono agent salvage therapy. The observed DCR was 42.9 and 21.1\%, with median TTP and median OS ranged from 91 days to 2.1 months and 414 days to 11.3 months, respectively. Yamaguchi et $\mathrm{al}^{135}$ conducted a study with 1-week on 1-week off regimen combining SL with bevacizumab for salvage therapy in heavily pretreated mCRC. Of the enrolled 31 patients, DCR was $65 \%(95 \% \mathrm{CI}$, $48 \%-100 \%)$ and response rate was 7\% (95\% CI, 0.7-\%22\%). The median PFS and OS were 5.3 (95\% CI, 2.1-9.3) and 9.9 (95\% CI, 7.4 to not available) months, respectively.

In the present study with 2 -week cycle of SL in heavily treated mCRC patients, DCR was observed at $61.1 \%$, which is close to the result of combining SL with bevacizumab at $65 \%$ of DCR. Although the median PFS at 2.55 months and OS at 7.63 months in the present study seemed numerically lower than those observed with SL/Bev study, this may be attributed to more patients with $\geq 5$ lines of prior therapies $(51.4 \%)$ in the current study than only $22 \%$ of patients with $\geq 4$ lines of prior therapies in the SL/Bev study.

The most common grade $3 / 4$ toxicities with $\mathrm{SL} / \mathrm{Bev}$ regimen were stomatitis/mucositis $(26 \%)$, diarrhea $(10 \%)$, anorexia ( $6 \%$ ), and neutropenia (3\%). Of note, grade 3 anemia $(6 \%)$ and febrile neutropenia $(3 \%)$ were also observed in the previous study, which were also consistent with frequently reported adverse effects of bevacizumab. In the present study, incidence of most frequent grade 3/4 toxicities was stomatitis/mucositis (14.6\%), bilirubin increased (4.9\%) and AST increased, anorexia, nausea, vomiting, fatigue, and hand-foot syndrome each with $2.4 \%$. No grade 3/4 diarrhea or hematological toxicities were observed. Compared with regorafenib and TAS-102, toxicity profiles of SL are more well-tolerated in refractory $\mathrm{mCRC}$ patients due to fewer grade 3 or higher hand -foot syndrome and neutropenia.

Our study demonstrated that no significant difference was observed in either disease stabilization or survival benefit between KRAS mutation status, which was seen in Yamaguchi et al's study. ${ }^{35}$

Based on the above studies, the 2-week cycle of S-1 plus calcium folinate, with 1-week on 1-week off regimen, had demonstrated comparable DCR, lower toxicities, along with other advantages including low cost and easy administration (oral route). We believe S-1 in combination with calcium folinate would be a good alternative with favorable risk-benefit profile for patients with heavily pretreated $\mathrm{mCRC}$. A further 
A

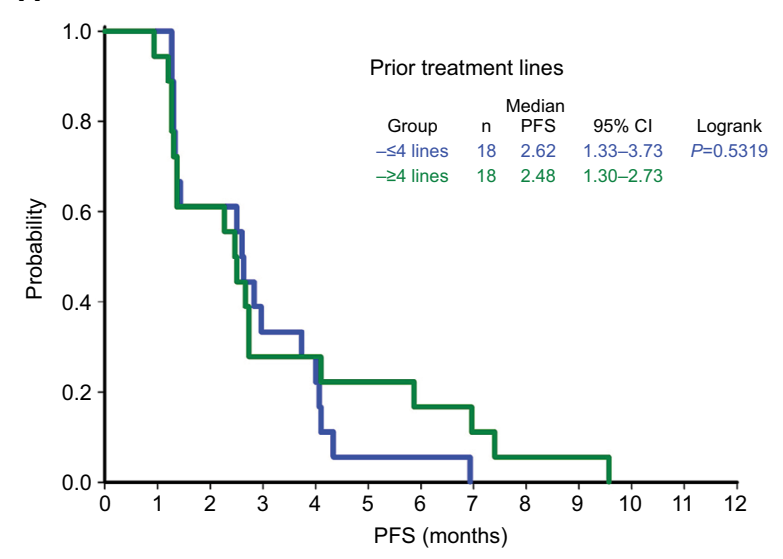

B

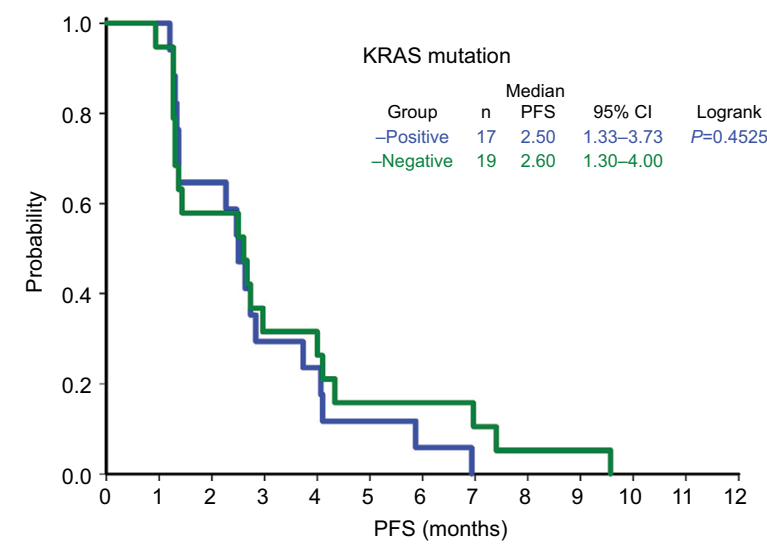

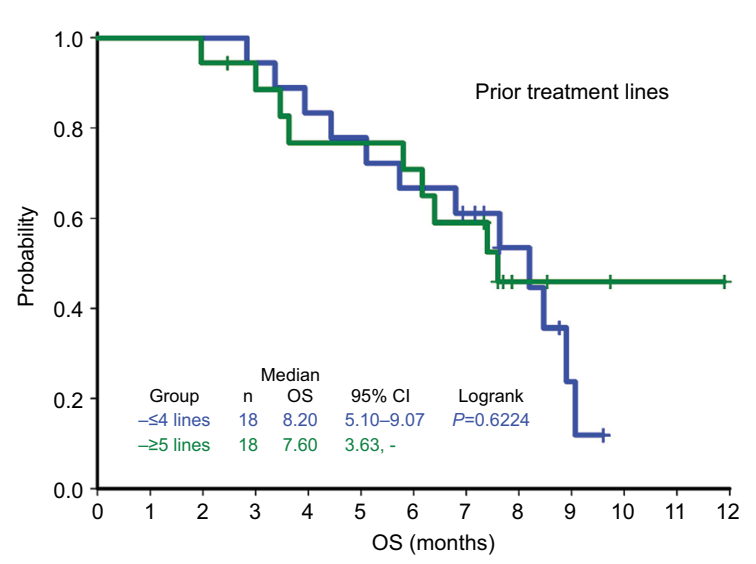

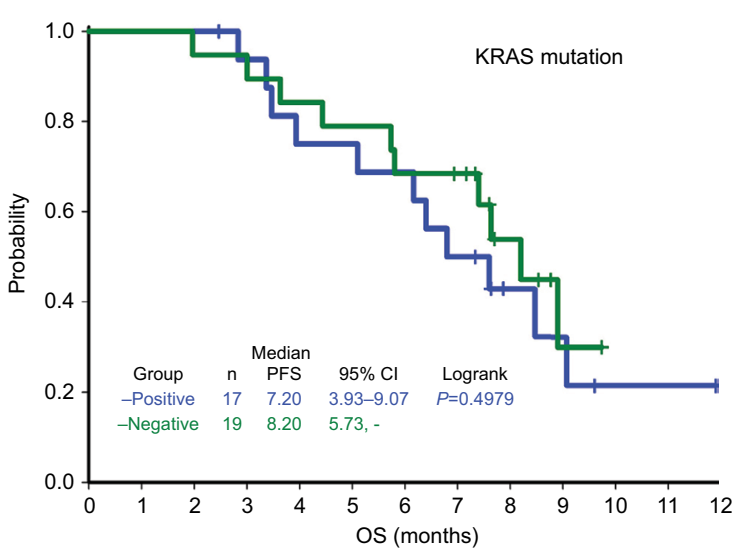

Figure 3 Subgroup analysis of Kaplan-Meier plot on PFS and OS according to numbers of prior treatment lines (A) or KRAS mutation status (B).

Note: Evaluation was assessed on evaluable population $(n=36)$.

Abbreviations: OS, overall survival; PFS, progression-free survival.

randomized control trial might be necessary to evaluate the usefulness of these current findings.

\section{Conclusion}

Fewer drugs are available for refractory mCRC. This is the first Phase II study to assess the efficacy and safety of salvage $\mathrm{SL}$ in heavily treated $\mathrm{mCRC}$. The results demonstrate that $\mathrm{SL}$ has potential as a salvage regimen in refractory $\mathrm{mCRC}$ patients especially in the heavily treated setting and is well tolerated in these patients.

\section{Availability of data and materials}

Individual deidentified participant data could be shared with others. All data such as specific data and study-related documents could also be shared. These datasets used and/or analyzed during the current study are available from the corresponding author on reasonable request after the results are published.

\section{Acknowledgment}

This study was sponsored by TTY Biopharm Company Ltd. (Taipei, Taiwan).

\section{Author contributions}

All authors contributed to data analysis, drafting or revising the article, gave final approval of the version to be published, and agree to be accountable for all aspects of the work.

\section{Disclosure}

The authors report no conflicts of interest in this work.

\section{References}

1. National Comprehensive Cancer Network [homepage on the Internet]. NCCN Clinical Practice Guidelines in Oncology: Colon Cancer, version 3.2014. Available from: http://www.nccn.org/professionals/physician_gls/f_guidelines.asp. Accessed May 20, 2014. 
2. Van Cutsem E, Cervantes A, Adam R, et al. ESMO consensus guidelines for the management of patients with metastatic colorectal cancer. Ann Oncol. 2016;27(8):1386-1422.

3. André T, Louvet C, Maindrault-Goebel F, et al. CPT-11 (irinotecan) addition to bimonthly, high-dose leucovorin and bolus and continuousinfusion 5-fluorouracil (FOLFIRI) for pretreated metastatic colorectal cancer. GERCOR. Eur J Cancer. 1999;35(9):1343-1347.

4. Jäger E, Heike M, Bernhard H, et al. Weekly high-dose leucovorin versus low-dose leucovorin combined with fluorouracil in advanced colorectal cancer: results of a randomized multicenter trial. Study Group for Palliative Treatment of Metastatic Colorectal Cancer Study Protocol 1. J Clin Oncol. 1996;14(8):2274-2279.

5. Falcone A, Ricci S, Brunetti I, et al. Phase III trial of infusional fluorouracil, leucovorin, oxaliplatin, and irinotecan (FOLFOXIRI) compared with infusional fluorouracil, leucovorin, and irinotecan (FOLFIRI) as first-line treatment for metastatic colorectal cancer: the Gruppo Oncologico Nord Ovest. J Clin Oncol. 2007;25(13):1670-1676.

6. Souglakos J, Androulakis N, Syrigos K, et al. FOLFOXIRI (folinic acid, 5-fluorouracil, oxaliplatin and irinotecan) vs FOLFIRI (folinic acid, 5-fluorouracil and irinotecan) as first-line treatment in metastatic colorectal cancer (MCC): a multicentre randomised phase III trial from the Hellenic Oncology Research Group (HORG). Br J Cancer. 2006;94(6):798-805.

7. Cassidy J, Clarke S, Díaz-Rubio E, et al. Randomized phase III study of capecitabine plus oxaliplatin compared with fluorouracil/folinic acid plus oxaliplatin as first-line therapy for metastatic colorectal cancer. $J$ Clin Oncol. 2008;26(12):2006-2012.

8. Petrelli N, Herrera L, Rustum Y, et al. A prospective randomized trial of 5-fluorouracil versus 5-fluorouracil and high-dose leucovorin versus 5-fluorouracil and methotrexate in previously untreated patients with advanced colorectal carcinoma. J Clin Oncol. 1987;5(10):1559-1565.

9. Wolmark N, Rockette H, Fisher B, et al. The benefit of leucovorinmodulated fluorouracil as postoperative adjuvant therapy for primary colon cancer: results from National Surgical Adjuvant Breast and Bowel Project protocol C-03. J Clin Oncol. 1993;11(10):1879-1887.

10. Cassidy J, Tabernero J, Twelves C, et al. XELOX (capecitabine plus oxaliplatin): active first-line therapy for patients with metastatic colorectal cancer. J Clin Oncol. 2004;22(11):2084-2091.

11. Porschen R, Arkenau HT, Kubicka S, et al. Phase III study of capecitabine plus oxaliplatin compared with fluorouracil and leucovorin plus oxaliplatin in metastatic colorectal cancer: a final report of the AIO Colorectal Study Group. J Clin Oncol. 2007;25(27):4217-4223.

12. Sargent DJ, Köhne CH, Sanoff HK, et al. Pooled safety and efficacy analysis examining the effect of performance status on outcomes in nine first-line treatment trials using individual data from patients with metastatic colorectal cancer. J Clin Oncol. 2009;27(12):1948-1955.

13. Chibaudel B, Tournigand C, Bonnetain F, et al. Therapeutic strategy in unresectable metastatic colorectal cancer: an updated review. Ther $A d v$ Med Oncol. 2015;7(3):153-169.

14. Lee DJ, Lee J, Lee HY, et al. Salvage S-1 monotherapy in metastatic colorectal cancer patients who failed irinotecan-based or oxaliplatinbased chemotherapy. Med Oncol. 2011;28(Suppl 1):291-294.

15. Jeung HC, Rha SY, Cho BC, et al. A phase II trial of S-1 monotherapy in metastatic colorectal cancer after failure of irinotecan- and oxaliplatincontaining regimens. Br J Cancer. 2006;95(12):1637-1641.

16. Kim SY, Hong YS, Kim BC, et al. A phase II study of S-1 plus irinotecan and oxaliplatin in heavily-treated patients with metastatic colorectal cancer. Invest New Drugs. 2009;27(3):269-274.

17. Muro K, Boku N, Shimada Y, et al. Irinotecan plus S-1 (IRIS) versus fluorouracil and folinic acid plus irinotecan (FOLFIRI) as second-line chemotherapy for metastatic colorectal cancer: a randomised phase $2 / 3$ non-inferiority study (FIRIS study). Lancet Oncol. 2010;11(9):853-860.

18. Thirion P, Michiels S, Pignon JP, et al. Modulation of fluorouracil by leucovorin in patients with advanced colorectal cancer: an updated meta-analysis. J Clin Oncol. 2004;22(18):3766-3775.
19. Tsukioka S, Uchida J, Tsujimoto H, et al. Oral fluoropyrimidine S-1 combined with leucovorin is a promising therapy for colorectal cancer: Evidence from a xenograft model of folate-depleted mice. Mol Med Rep. 2009;2(3):393-398.

20. Koizumi W, Boku N, Yamaguchi K, et al. Phase II study of S-1 plus leucovorin in patients with metastatic colorectal cancer. Ann Oncol. 2010;21(4):766-771.

21. Ohtsu A, Baba H, Sakata Y, et al. Phase II study of S-1, a novel oral fluoropyrimidine derivative, in patients with metastatic colorectal carcinoma. Br J Cancer. 2000;83(2):141-145.

22. Grothey A, Van Cutsem E, Sobrero A, et al. Regorafenib monotherapy for previously treated metastatic colorectal cancer (CORRECT): an international, multicentre, randomised, placebo-controlled, phase 3 trial. Lancet. 2013;381(9863):303-312.

23. Li J, Qin S, Xu R, et al. Regorafenib plus best supportive care versus placebo plus best supportive care in Asian patients with previously treated metastatic colorectal cancer (CONCUR): a randomised, double-blind, placebo-controlled, phase 3 trial. Lancet Oncol. 2015;16(6):619-629.

24. Mayer RJ, Van Cutsem E, Falcone A, et al. Randomized trial of TAS-102 for refractory metastatic colorectal cancer. $N$ Engl J Med. 2015;372(20):1909-1919.

25. Goldberg RM, Montagut C, Wainberg ZA, et al. Optimising the use of cetuximab in the continuum of care for patients with metastatic colorectal cancer. ESMO Open. 2018;3(4):e000353.

26. Santini D, Vincenzi B, Addeo R, et al. Cetuximab rechallenge in metastatic colorectal cancer patients: how to come away from acquired resistance? Ann Oncol. 2012;23(9):2313-2318.

27. Rossini D, Santini D, Cremolini C, et al. PD-026 Rechallenge with cetuximab + irinotecan in 3rd-line in RAS and BRAF wild-type metastatic colorectal cancer $(\mathrm{mCRC})$ patients with acquired resistance to 1st-line cetuximab+irinotecan: The phase II CRICKET study by GONO. Ann Oncol. 2017;28(Suppl 3):iii1-iii12.

28. Douillard JY, Hoff PM, Skillings JR, et al. Multicenter phase III study of uracil/tegafur and oral leucovorin versus fluorouracil and leucovorin in patients with previously untreated metastatic colorectal cancer. J Clin Oncol. 2002;20(17):3605-3616.

29. Carmichael J, Popiela T, Radstone D, et al. Randomized comparative study of tegafur/uracil and oral leucovorin versus parenteral fluorouracil and leucovorin in patients with previously untreated metastatic colorectal cancer. J Clin Oncol. 2002;20(17):3617-3627.

30. Lembersky BC, Wieand HS, Petrelli NJ, et al. Oral uracil and tegafur plus leucovorin compared with intravenous fluorouracil and leucovorin in stage II and III carcinoma of the colon: results from National Surgical Adjuvant Breast and Bowel Project Protocol C-06. J Clin Oncol. 2006;24(13):2059-2064.

31. Li J, Xu R, Xu J, et al. Phase II study of S-1 plus leucovorin in patients with metastatic colorectal cancer: Regimen of 1 week on, 1 week off. Cancer Sci. 2017;108(10):2045-2051.

32. Fukushima M, Fujioka A, Uchida J, Nakagawa F, Takechi T. Thymidylate synthase (TS) and ribonucleotide reductase (RNR) may be involved in acquired resistance to 5-fluorouracil (5-FU) in human cancer xenografts in vivo. Eur J Cancer. 2001;37(13):1681-1687.

33. Murakami Y, Kazuno H, Emura T, Tsujimoto H, Suzuki N, Fukushima M. Different mechanisms of acquired resistance to fluorinated pyrimidines in human colorectal cancer cells. Int $J$ Oncol. 2000;17(2):277-283.

34. Shirasaka T, Nakano K, Takechi T, et al. Antitumor activity of $1 \mathrm{M}$ tegafur-0.4 M 5-chloro-2,4-dihydroxypyridine-1 M potassium oxonate (S-1) against human colon carcinoma orthotopically implanted into nude rats. Cancer Res. 1996;56(11):2602-2606.

35. Yamaguchi K, Taniguchi H, Komori A, et al. A single-arm phase II trial of combined chemotherapy with S-1, oral leucovorin, and bevacizumab in heavily pre-treated patients with metastatic colorectal cancer. $B M C$ Cancer. 2015;15:601. 


\section{Supplementary material}
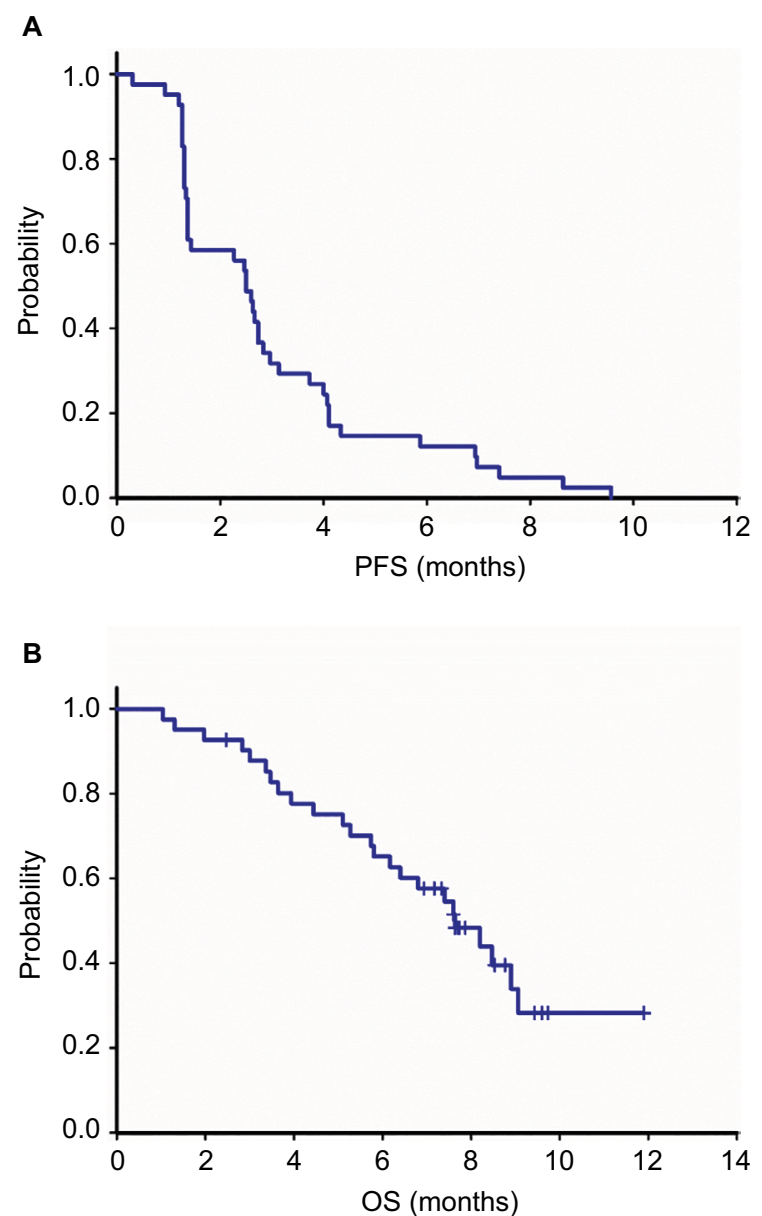

Figure SI Kaplan-Meier survival curve for PFS (A) and OS (B) in the ITT population $(n=4 I)$.

Notes: The median PFS was 2.50 months ( $95 \% \mathrm{Cl}, \mathrm{I} .37-2.83)$. The median OS was 7.63 months (95\% Cl, 5.80-9.07).

Abbreviations: ITT, intend-to-treat; OS, overall survival; PFS, progression-free survival.

\section{Publish your work in this journal}

Cancer Management and Research is an international, peer-reviewed open access journal focusing on cancer research and the optimal use of preventative and integrated treatment interventions to achieve improved outcomes, enhanced survival and quality of life for the cancer patient. The manuscript management system is completely online and includes

a very quick and fair peer-review system, which is all easy to use. Visit http://www.dovepress.com/testimonials.php to read real quotes from published authors. 